\title{
Re-Educating Residents About Non-Invasive Colorectal Cancer Screening: An Approach to Improving Colon Cancer Screening Compliance
}

\author{
Gabriel Melki ${ }^{\mathrm{a}, \mathrm{c}}$, Moutaz Ghrewati ${ }^{\mathrm{a}}$, Hadir Mohamed ${ }^{\mathrm{a}}$, Shaker Barham ${ }^{\mathrm{b}}$, Ashima Kapoor ${ }^{\mathrm{a}}$, \\ Farhan Ayoub ${ }^{\mathrm{a}}$, Abdalla Mohamed ${ }^{\mathrm{a}}$, Alexander $\mathrm{Wu}^{\mathrm{a}}$, Sugabramya Kuru ${ }^{\mathrm{a}}$, Alisa Farokhian ${ }^{\mathrm{a}}$, \\ Rana Garris ${ }^{\mathrm{a}}$, Chandra Chandran ${ }^{\mathrm{a}}$, Matthew Grossman ${ }^{\mathrm{b}}$, Walid Baddoura ${ }^{\mathrm{b}}$
}

\begin{abstract}
Background: Colorectal cancer is the third leading cause of cancer death; therefore early detection by screening is beneficial. Residents at a clinic in NJ, USA were not offering other forms of colon cancer screening when patients refused colonoscopy, which lead to the creation of the quality improvement project.
\end{abstract}

Methods: Residents practicing at the clinic were given an anonymous survey determining which method of colon cancer screening they used and which alternative method they offered when patients refused the original method. The residents were educated about all methods of colon cancer screening and the residents were resurveyed.

Results: A total of $64 \%$ of residents offered less invasive testing when colonoscopy was refused. Six months after education, $95 \%$ of residents offered less invasive testing when colonoscopy was refused.

Conclusions: Early detection and removal of polyps by colonoscopy reduce the risk of cancer development. Colonoscopy is the gold standard for colon cancer screening; however other less invasive modalities are approved. This quality improvement project lead to offering the fecal immunochemical test or fecal occult blood test once patients refused colonoscopy at the clinic, increasing the number of patients receiving colorectal cancer screening, and thus providing better medical care.

Keywords: Colon cancer; Colon cancer screening; Fecal occult blood test; Quality improvement

\footnotetext{
Manuscript submitted July 31, 2019, accepted September 6, 2019

aDepartment of Internal Medicine, St. Joseph's University Medical Center, Paterson, NJ, USA

bepartment of Gastroenterology, St. Joseph's University Medical Center, Paterson, NJ, USA

${ }^{\mathrm{c} C o r r e s p o n d i n g ~ A u t h o r: ~ G a b r i e l ~ M e l k i, ~ D e p a r t m e n t ~ o f ~ I n t e r n a l ~ M e d i c i n e, ~ S t . ~}$ Joseph's University Medical Center, Paterson, NJ, USA.

Email: gabriel.melki@mail.mcgill.ca
}

doi: https://doi.org/10.14740/gr1205

\section{Introduction}

Colorectal cancer (CRC) is the third leading cause of cancer death [1], and therefore early detection by screening is beneficial [2]. Residents in primary care clinics offer screening modalities for $\mathrm{CRC}$ screening starting at the age of 50. At a clinic in NJ, USA, the primary method of CRC screening used was colonoscopy every 10 years starting at the age of 50 ; however when patients refused colonoscopies, other less invasive testing were not being offered. This prompted the investigators to intervene and assess the percentage of residents not offering less invasive testing. Residents were re-educated about alternate screening modalities for CRC and residents were reassessed.

\section{Materials and Methods}

Sixty-three residents were given survey (Supplementary Material 1, www.gastrores.org) based on multiple choice questionnaires, which asked first test used for CRC screening and what they would do if their first screening test was refused (if invasive). The questionnaire was anonymous. Results were obtained to assess the percentage of residents offering less invasive testing. Residents were re-educated about all methods of CRC screening that are approved as of 2018 [3]. At 6 months, the residents were given the questionnaire and results were used to compare the percentage of residents offering less invasive testing. Statistical methods used to analyze the study were the percent change and the percent difference. All analyses were performed using Microsoft Office Excel 2007 (Microsoft Corp., Redmond, WA, USA). The Institutional Review Board approved this study, which was conducted in compliance with the ethical standards of the responsible institution on human subjects.

\section{Results}

At this institution, the first test used for CRC screening was colonoscopy at $73 \%$, followed by fecal occult blood test (FOBT) at $19 \%$ (Fig. 1). A total of $64 \%$ of residents were offered less invasive testing when colonoscopy was refused, usually fecal immunochemical test (FIT) (Fig. 2). After 6 months of resident re- 


\section{What Method of Screening are Residents offering?}

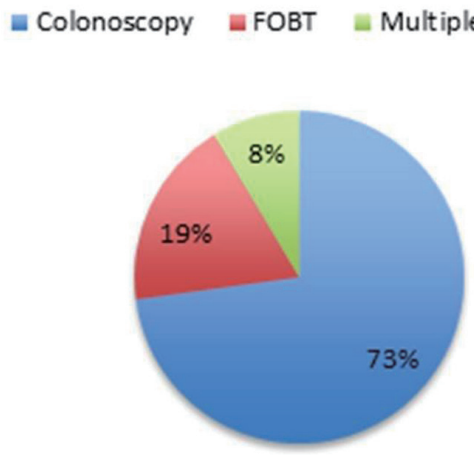

Figure 1. Pie chart revealing what residents offer as a primary mode of screening.

education, colonoscopy was still the most used at 73\%. A total of $95 \%$ residents offered less invasive testing when colonoscopy was refused (Fig. 3). The percent change revealed an increase of $48.4375 \%$ in terms of residents offering less invasive testing when patients refused colonoscopy. The percent difference indicated a difference of action from the residents of $38.9937 \%$.

\section{Discussion}

Most cases of CRC develop from polyps; therefore early detection and removal by colonoscopy reduce the risk of CRC development [1-3]. Colonoscopy is the gold standard for CRC screening; however other modalities are also approved such as
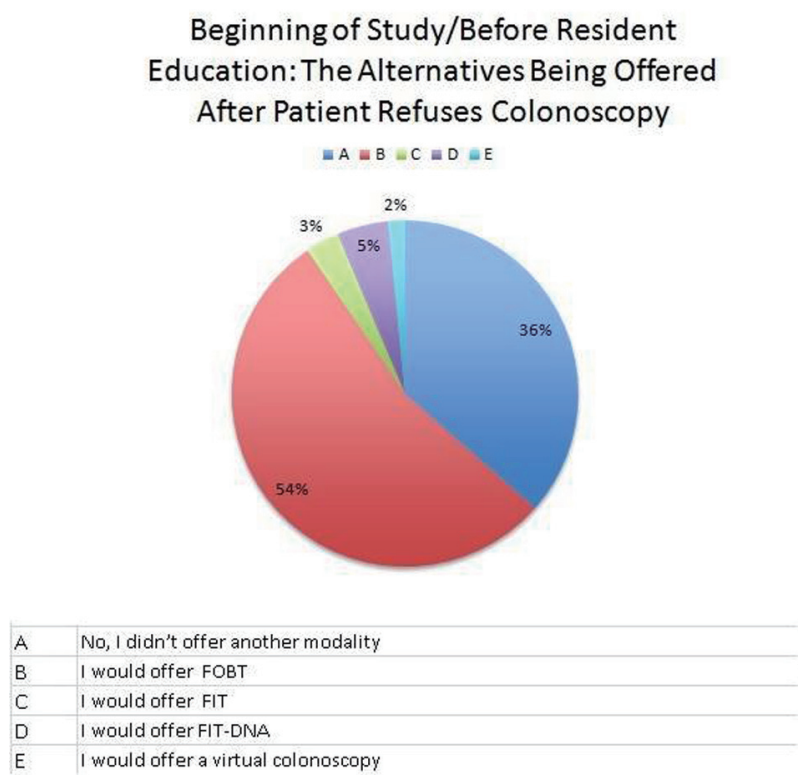

Figure 2. A pie graph at the beginning of the study focusing on what residents offer when a patient refuses colonoscopy (alternative less invasive procedures).

\section{End of Study/Six Months Post Resident Education: The Alternatives Being Offered After Patient Refuses Colonoscopy

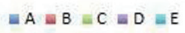

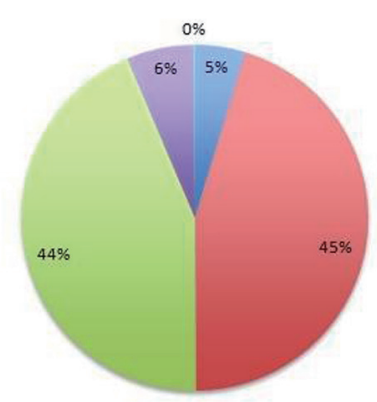

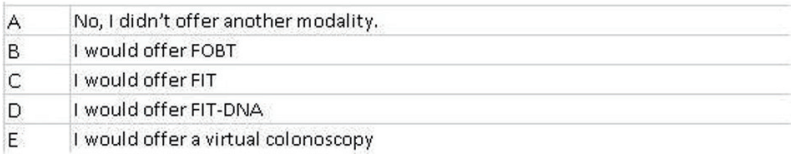

Figure 3. Pie graph at the end of the study focusing on what residents offer when a patient refuses colonoscopy (alternative less invasive procedures).

FOBT test yearly, and it is important to offer these modalities when colonoscopies are refused [1-3]. It was noticed that residents were not offering these test when patients refused colonoscopies and this is why this quality improvement (QI) project was created. After 6 months of re-education, residents went from offering less invasive testing once refusal of colonoscopy from $64 \%$ to $95 \%$, revealing a percent change of $48.4375 \%$ in terms of residents offering less invasive testing when patients refused colonoscopy. The data of the study were gathered from an anonymous survey issued before and 6 months after resident re-education. At the time of the study, the facility only had paper charts. Relying on resident's response on a survey is less ideal than analyzing medical chart work in order to determine what was offered to the patients for CRC screening and what was offered to the patient when they refused colonoscopy. This is a limitation of the study. Future studies could focus on documented refusal and alternative tests offered by looking through an electronic medical record. However, after re-education, residents reported that they were offering less invasive testing (such as FOBT or FIT) when patients refused colonoscopy. The attending preceptors at the institution realized this and are now offering the test automatically when patients refuse colonoscopy. This would increase the number of patients screened. Patients could have many reasons to refuse a screening colonoscopy including thinking they do not need it since they have no symptoms or CRC risk factors, discomfort with bowel prep for colonoscopy and discomfort with the invasive procedure itself [4]. By offering FOBT or FIT for screening when patients refuse colonoscopy, the patients that refuse colonoscopy due to invasive testing, discomfort with procedure and bowel prep regimen will be screened using noninvasive methods. If fecal tests are positive, at a follow-up visit, a discussion is made about having a colonoscopy. Patients generally agree to colonoscopy when noninvasive screening tests are abnormal. Unfortunately, 
some patients refuse to undergo a colonoscopy despite a positive FIT for various reasons but mostly related to procedural discomfort. For these patients, we recommend re-educating them at every visit about the benefits of CRC screening, the possibility of curing colon cancer when detected early or preventing it by resecting polyps with malignant potential [2].

This QI project aims to improve compliance with CRC screening using FIT or FOBT once patients refuse colonoscopy. Our intervention with re-educating primary care clinic medical staff resulted in improvement in screening compliance. This ultimately means providing better medical care.

\section{Supplementary Material}

Supplementary Material 1. Colorectal Cancer Screening Survey.

\section{Acknowledgments}

We thank Dr. Patrick Michael (Program Director of the Internal Medicine Residency), Dr. Michael Agnelli (Associate Program Director of the Internal Medicine Residency), Dr. Monisha Singhal (Associate Program Director of the Internal Medicine Residency) and Dr. Robert Lahita (Chairman of the Department of Medicine) for their continuous support and guidance.

\section{Financial Disclosure}

No funding was provided to any of the authors.

\section{Conflict of Interest}

The authors declare that they have no conflict of interest.

\section{Informed Consent}

Informed consent was obtained for this research article.

\section{Author Contributions}

GM was the principle investigator, who contributed to project conception/design, manuscript preparation and writing, survey creation, methods, survey administration, data collection and analysis. MG, HM, AK, FA, AM and AW contributed to survey creation, and data collection. SB was involved in manuscript writing and editing, and mentorship. SK, AF and $\mathrm{RG}$ contributed to technical methods and editing. $\mathrm{CC}$ and $\mathrm{MG}$ were the supervising attending physicians, playing a role in mentorship and guidance. WB was the supervising attending physicians, playing a role in mentorship guidance and manuscript editing. All authors reviewed the final manuscript.

\section{References}

1. Issa IA, Noureddine M. Colorectal cancer screening: An updated review of the available options. World J Gastroenterol. 2017;23(28):5086-5096.

2. Freeman HJ. Early stage colon cancer. World J Gastroenterol. 2013;19(46):8468-8473.

3. US Preventive Services Task Force, Bibbins-Domingo K, Grossman DC, Curry SJ, Davidson KW, Epling JW, Jr., Garcia FAR, et al. Screening for colorectal cancer: US Preventive Services Task Force Recommendation Statement. JAMA. 2016;315(23):2564-2575.

4. Denberg TD, Melhado TV, Coombes JM, Beaty BL, Berman K, Byers TE, Marcus AC, et al. Predictors of nonadherence to screening colonoscopy. J Gen Intern Med. 2005;20(11):989-995. 